\title{
Corela
}

Cognition, représentation, langage

17-2 | 2019

Vol. $17, \mathrm{n}^{\circ} 2$

\section{La distinction accompli/inaccompli dans le récit en FLE : enjeux pour la conception de matériel pédagogique.}

Loïc Renoud

\section{OpenEdition}

Journals

Édition électronique

URL : http://journals.openedition.org/corela/9828

DOI : $10.4000 /$ corela. 9828

ISSN : 1638-573X

Éditeur

Cercle linguistique du Centre et de l'Ouest - CerLICO

\section{Référence électronique}

Loïc Renoud, «La distinction accompli/inaccompli dans le récit en FLE : enjeux pour la conception de matériel pédagogique. », Corela [En ligne], 17-2 | 2019, mis en ligne le 08 janvier 2021, consulté le 21 janvier 2021. URL : http://journals.openedition.org/corela/9828 ; DOI : https://doi.org/10.4000/corela. 9828

Ce document a été généré automatiquement le 21 janvier 2021.

\section{cc) (i) (3)}

Corela - cognition, représentation, langage est mis à disposition selon les termes de la licence Creative Commons Attribution - Pas d'Utilisation Commerciale - Partage dans les Mêmes Conditions 4.0 International. 


\title{
La distinction accompli/inaccompli dans le récit en FLE : enjeux pour la conception de matériel pédagogique.
}

\author{
Loïc Renoud
}

Cette recherche a reçu le soutien financier de la Société Japonaise pour la Promotion de la Science (JSPS-KAKENHI $n^{\circ}$ JP18K12452).

\section{Introduction}

1 Il est admis que le système de la temporalité verbale est une difficulté d'apprentissage du Français Langue Étrangère (FLE), en particulier la relation entre l'imparfait et le passé composé (Courtillon, 1981, 2001 ; Dansereau, 1987 ; de Salins, 1996 ; Bergström, 1997). Cette relation est le sujet de cet article, qui traitera en particulier de la conception de matériel pédagogique pour l'apprentissage de ces temps et de sa mise en œuvre auprès de publics endolingues initiaux en situation guidée.

Différents facteurs jouent dans le développement de la morphologie verbale. L'« hypothèse de l'aspect " d'une part et la nécessité de l'organisation temporelle du récit d'autre part sont deux facteurs "internes " à l'emploi de la langue enseignée (Véronique et al, 2009), c'est-à-dire indépendants de la langue de départ et de l'approche pédagogique. L'hypothèse de l'aspect (Andersen \& Shirai, 1994) prévoit que les procès atéliques soient plus souvent associés à un temps imperfectif, et les procès téliques à un temps perfectif. Dans une étude basée sur un récit filmique réalisée avec des étudiants (États-Unis) aux $2^{2 \mathrm{ème}}, 4^{\text {ème }}$ et $6^{\text {ème }}$ semestres de leurs études de français, Bergström (1997) montre que la tendance se vérifie en FLE. On peut représenter cette tendance de la façon suivante (tableau 1) : 


\begin{tabular}{|c|c|c|c|}
\hline état & activité & accomplissement & achèvement \\
\hline il a été & & $\begin{array}{l}\text { il a repassé } \\
\text { chemise }\end{array}$ & il a ouvert la porte \\
\hline il a été & il a chanté & $\begin{array}{l}\text { il a repassé } \\
\text { chemise }\end{array}$ & il a ouvert la porte \\
\hline il était & il chantait & $\begin{array}{l}\text { il repassait } \\
\text { chemise }\end{array}$ & il ouvrait la porte \\
\hline il était & il chantait & $\begin{array}{l}\text { il repassait } \\
\text { chemise }\end{array}$ & il ouvrait la porte \\
\hline
\end{tabular}

Tableau : tendances de l'acquisition de l'imparfait et du passé composé selon l'hypothèse de l'aspect (d'après Bergström (1997), nos exemples).

Les cases encadrées représentent les premiers emplois apparaissant dans l'interlangue d'après l'hypothèse de l'aspect, et les flèches l'ordre d'acquisition probable (le sens de l'imparfait dans «il ouvrait la porte" n'est pas compris par les apprenants qui débutent, etc.). Toutefois, Bergström (1997) rapporte que le passé composé a souvent été employé sur des procès d'activité.

4 Le second facteur interne correspond à la contrainte de devoir organiser les relations temporelles entre les procès pour l'intelligibilité de son récit. En effet, Weinrich (1973) montre que la parole ancrée dans la situation de l'échange s'appuie pragmatiquement sur cette situation qui en est comme l'arrière-plan par défaut. En revanche, le « monde raconté » du récit est tributaire des seuls moyens linguistiques pour la mise en relief des actions devant un arrière-plan (p. 116). Les qualités proprement perceptives de cette opposition de plan nous semblent également importantes. Au premier plan, les procès successifs constitueront une chaîne d'actions qui se détache d'un fond dont ces actions dépendent. À l'arrière-plan sont indiquées les causes et modalités des actions (Reinhart, 1984). Pour leur part, Andersen et Shirai (1994) remarquent que dans les premières interlangues, les verbes reçoivent des marques d'accompli au premier plan tandis que les verbes pour des états ou actions indiquant les circonstances ne sont pas fléchis. D'après les auteurs, ces états ou actions sont traités comme des caractéristiques des environnements ou personnes évoqués et sont sentis comme se prolongeant audelà de la durée pendant laquelle l'action de premier plan a eu lieu. Elles ne finissent pas avec les actions du premier plan (Andersen \& Shirai, 1994 : 150). En d'autres termes, les procès de l'arrière-plan sont traités comme simultanés à ceux du premier plan.

5 La langue de départ et l'approche pédagogique influent également, en particulier sur le rythme d'acquisition. Différentes études semblent montrer que des formes dans l'interlangue résultent des essais par les apprenants de reconstituer des caractéristiques de la langue de départ qui n'existent pas ou qui sont marquées différemment dans la langue enseignée (Véronique et al., 2009 : 194-204). Par ailleurs, Besse (2005) note que, le cas échéant en fonction des contextes de scolarisation, les catégories métalinguistiques pour l'apprentissage de la langue de départ et de la ou des langues étrangères ultérieurement apprises avant le français (il s'agit surtout, pour les non anglophones, de l'anglais) étaient réutilisées par les apprenants. Toutefois, dans la mesure où l'on s'intéresse au contexte endolingue, donc avec des apprenants de diverses langues de départ, on n'avancera pas plus loin ces questions. En revanche, en nous focalisant sur la relation entre l'imparfait et le passé composé, on veut explorer un aspect essentiel de l'approche d'enseignement, l'influence du matériel pédagogique 
mis en œuvre. Comment le matériel et l'utilisation qu'il suppose peuvent-ils soutenir l'apprentissage et développer la maitrise de ces deux temps dans le récit?

6 Il y a au moins deux types de réponses possibles. Dans le premier, il est montré que le verbe conjugué à l'imparfait ou au passé composé correspond à une "perspective humaine incarnée» (Tomasello, [2003] 2005: 53) sur le procès, laquelle sert à l'organisation temporelle des plans du récit (succession des procès au premier plan, simultanéité à l'arrière-plan). Le matériel pédagogique existant tend vers ce type de réponse, à divers degrés. Dans le deuxième, on voudrait montrer que cette perspective s'élabore dans la syntaxe de l'énoncé avec le verbe conjugué. En conséquence, on suggèrerait que c'est à partir de la syntaxe de l'énoncé que les deux plans de l'organisation narrative du récit, assez universelle, devraient être pratiqués avec les apprenants débutants. Cette proposition, qui s'inscrit dans le cadre de la linguistique énactive, sera décrite en fin d'article.

7 Auparavant, la section suivante s'intéresse aux enjeux didactiques du premier type de réponse. On reprendra des éléments de discussion ces vingt dernières années, où sont souvent mis en cause la qualité du matériel et le bien fondé des démarches. Doit-on privilégier une approche basée sur la distinction aspectuelle ou bien sur le rôle des temps dans le récit? Pour un aperçu concret du matériel existant, la section 3 donnera une vue d'ensemble des descriptions grammaticales de la relation entre le passé composé et l'imparfait dans 17 manuels de FLE généralistes publiés entre 2006 et 2016, puis s'attachera plus particulièrement à décrire l'approche d'un manuel la plus riche à notre sens.

\section{L'enseignement et l'apprentissage de la distinction entre l'imparfait et le passé composé dans le récit : des enjeux didactiques discutés.}

\subsection{Démarche basée sur la distinction aspectuelle}

Différents auteurs ont plaidé pour que la distinction aspectuelle entre inaccompli et accompli ${ }^{1}$ constitue la base de l'approche (Courtillon, 1985, 2001 ; Dansereau, 1987 ; de Salins, 1997), ou soit systématiquement mise en relation en relation avec les listes d'emploi (Judge, 2002), ou encore pour rappeler que la visée sur le procès est un choix énonciatif (Beacco, 2010).

Dansereau (1987) observe que les manuels pour les niveaux initiaux masquent la réelle distinction entre les deux temps, qui est d'ordre aspectuel. L'auteure relève différentes catégories de problèmes dans le matériel traditionnel, liés à une simplification excessive dans la transposition didactique, à des confusions terminologiques, voire des erreurs théoriques, et à l'emploi de la première langue dans la traduction des verbes conjugués. On peut reformuler ces critiques de la façon suivante :

10 - L'opposition achevé/inachevé induit en erreur sans mention du point de référence dans le passé (notion absente des manuels), car les actions à l'imparfait sont certes montrée inachevées mais suivant la situation, et à plus forte raison dans le récit, on infère qu'elles le sont au moment de l'énonciation. Si la précision n'est pas faite, l'apprenant peut donc objecter que le procès à l'imparfait est achevé. 
11 - Les notions de durée et continuité sont ambigües, car le temps dans l'intervalle borné d'un «événement " (« event » au sens de Mourelatos, 1978: 433) non ponctuel peut sembler continu. De même, "action à un moment précis " pour expliquer le passé composé entrera en contradiction avec la vision sécante de l'imparfait de second plan, qui est instantanée. Il s'agit à nouveau d'un problème lié au point de référence ou plutôt à l'absence de la notion dans l'explication pédagogique.

- Les termes d'“état » et de "description » pour caractériser l'imparfait sont approximatifs : l'apprenant se demandera pourquoi «j'ai eu peur » n'est pas un état, pourquoi « elle a fermé la porte » ne décrit pas un action.

- La fréquence n'est pas une propriété de l'imparfait ${ }^{2}$, or les exemples systématiques de l'imparfait avec des marqueurs temporels de fréquence mettent en place des associations automatiques qui freinent la compréhension du sens aspectuel de l'imparfait ${ }^{3}$.

- Les composantes de l'énoncé interagissent différemment dans chaque langue. Il vaut mieux laisser de côté la première langue : Le dimanche, j'allais (went) à l'église. Dimanche, je suis allé(e) (went) à l'église.

12 Ce constat a poussé l'auteure à recourir dans sa classe (public anglophone, niveaux initiaux) à une approche basée sur la distinction d'aspect, mais dont la formulation est, à notre sens, plus opératoire que conceptuelle : "The imparfait is used to relate what the conditions were / what was going on ("Quelles étaient les conditions?») and the passé composé to relate what happened / what happened next ("Qu'est-ce qui s'est passé? ») ». (Dansereau, 1987: 37) Dans la mise en œuvre, l'auteure dit y revenir dès qu'un emploi des apprenants le nécessite dans une situation d'apprentissage donnée. (focalisation incidente sur la forme). Dansereau préconise l'emploi d'exemples nets montrant la simultanéité de deux actions, avec l'imparfait dans la principale et le passé composé dans une subordonnée avec « quand $»^{4}$. Finalement, l'approche que Dansereau (1985) met en place, c'est-à-dire en revenant au même principe opératoire (mentionné cidessus) au gré des situations, évoque la façon dont procède Judge (2002). En revanche, Judge, qui certes traite du cas de niveaux avancés, dit expliquer franchement les valeurs d'aspect pour gloser les emplois rencontrés en classe (2002 : 148).

De même, de Salins (1996) prend clairement position pour une approche «aspectuelle». La citation suivante est extraite d'un manuel de cette auteure à l'attention des étudiants des formations en didactique du FLE. Le parti pris de de Salins est une perspective onomasiologique (préface), dans la droite ligne de l'approche fonctionnelle-notionnelle (Courtillon, 1985, voir infra). Mais le manuel comporte aussi une dimension pratique, rapportant des erreurs d'apprenants souvent commises, suggérant parfois des démarches. Ici, l'auteure veut montrer l'intérêt d'une approche subjective de l'alternance entre l'imparfait et le passé composé. On entend par là une démarche pour inculquer les moyens de choisir le temps suivant sa "vision » du procès, et non pas d'après l'aspect lexical ou d'autres indices contextuels. L'exemple pris est celui de la durée. Dans la citation, on remarquera aussi la gestuelle suggérée par l'auteure pour illustrer l'aspect grammatical, qui ne nous semble pas aller de soi. À notre sens, les gestes devraient souligner l'expérience de la progression du sens dans l'énoncé et l'émergence du rôle du sujet tel qu'il y est induit (sur cela, voir notre proposition section 4):

14 Les temps du passé (imparfait/passé composé) seraient peut-être plus clairement compris si on expliquait qu'il y a, en français, deux moyens d'évoquer le procès du 
verbe, de même qu'il existe parfois deux termes pour exprimer un même concept : an et année, matin et matinée, soir et soirée. On admettra sans trop de difficulté, que la réalité à laquelle renvoie le premier de ces termes ne dure pas moins longtemps que l'autre! Ce qui les différencie l'un de l'autre ce n'est pas la durée, c'est la vision tensive (an) ou extensive (année) d'une même réalité. Pour présenter de façon imagée la différence entre imparfait et passé composé, l'enseignant pourrait aussi évoquer gestuellement la forme d'un accordéon : déplié $=$ extensif $=$ imparfait $/$ plié $=$ tensif $=$ passé composé. (de Salins, 1996 : 157)

L'approche fonctionnelle-notionnelle pour la grammaire a été promue par Janine Courtillon, qui co-écrira la série de manuels Libre Échange avec Geneviève-Dominique de Salins. On se penchera brièvement sur cette approche, de laquelle le manuel de formation de de Salins (1996) peut être rapproché. Ensuite, on pourra observer comment ces principes se traduisent dans le manuel Libre Échange.

D'après Courtillon (1985), la construction du sens dans une nouvelle langue est facilitée par l'exposition à des données linguistiques organisées suivant les notions de la langue. Les notions sont des composantes sémantiques tendant à être universelles (en particulier spatiales pour l'intériorité / l'extériorité, et temporelles pour les rapports d'antériorité, simultanéité, postériorité, etc.). Il y a un domaine conceptuel humain commun, graduellement discrétisé dans les signifiés différents des différentes languescultures et à l'œuvre dans les fonctions communicatives. Pottier est cité comme une source influente. Dans la didactique du FLE, une démarche pour le passé composé et l'imparfait suivant cette perspective (cf. Courtillon, 1985 : 37-39) présenterait d'abord le passé composé et l'imparfait dans leurs fonctions sans explication grammaticale, respectivement de rapport d'actions passées et de description d'états passés. Ensuite, l'imparfait serait contrasté avec le plus-que-parfait. Enfin seulement serait abordée la distinction aspectuelle entre l'imparfait et le passé composé sur des procès statiques (j'étais... / j'ai été..., je pensais... / j'ai pensé..., je croyais... / j'ai cru...) mettant en jeu une différence possible de perspective qui par exemple en anglais ou en allemand n'est pas reflétée dans la morphologie verbale. Pour la démarche, il s'agit de répéter l'exposition à des données où la distinction est justifiée fonctionnellement : « je n'ai pas répondu au téléphone parce que... je ne pouvais pas (état : j’étais occupée) / je n'ai pas pu (résultat: je suis arrivée trop tard)». Dans le cadre théorique élaboré par cette auteure, « état » et « résultat » sont des notions.

\section{2. Libre Échange : exemple d'une approche aspectuelle}

17 Dans la structure de la leçon, le manuel Libre Échange (2001) est typique de l'approche communicative d'avant le Cadre Européen Commun de Référence pour les Langues (CECRL). La partie compréhension de cette leçon avec les deux temps comprend un dialogue introducteur (titré: "J'ai passé une bonne soirée.»), un repérage des temps, des exercices sur le passé composé et l'imparfait séparément et une réflexion sur l'emploi des temps. La phase de production comprend des jeux de rôles, et des exercices guidés sur la combinaison des deux temps (fait au passé composé, explication à l'imparfait). Le tableau 2 ci-dessous reproduit la présentation grammaticale des deux temps qui vient après :

Le passé composé et l'imparfait 


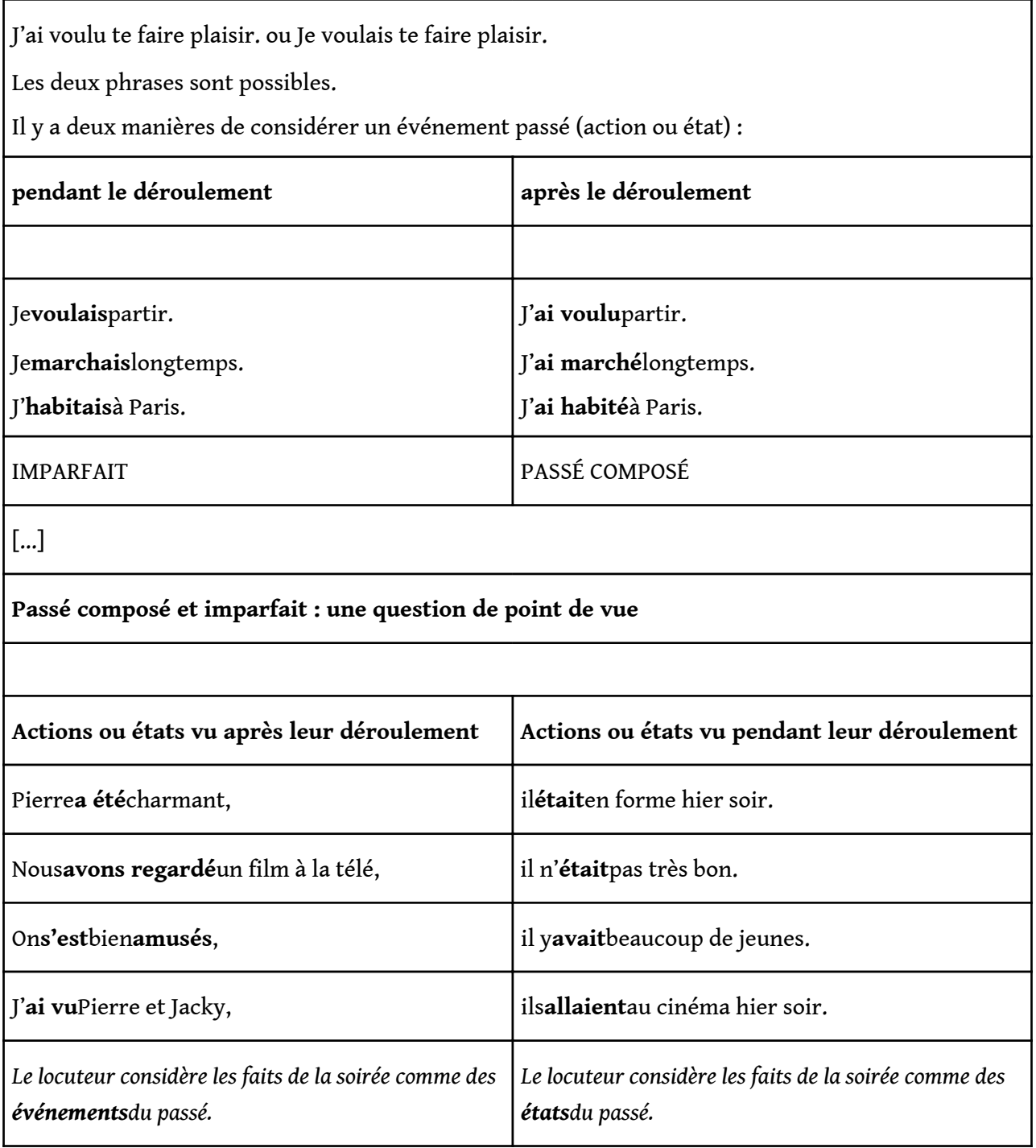

Tableau : le passé composé et l'imparfait dans Libre Échange 1 (p. 171), exemple d'une approche aspectuelle.

Le principe est clairement onomasiologique. Une «manièr[e] de considérer un événement passé » se matérialise en la forme adéquate: les termes «imparfait » et "passé composé » viennent après la définition et les exemples. Ensuite, le cœur de la distinction concerne la «saisie aspectuelle » de l'événement, à la façon de Pottier, pendant ou après son déroulement. Ultérieurement, Pottier (2001) résumera qu'« $[\mathrm{u}] \mathrm{n}$ événement est surtout perceptible quand il se déroule, et dans son terme et ses suites. » (p. 83) L'entrée dans la relation entre les deux temps est le choix de visée, abstraction faite du sémantisme du procès, en exposant dès l'abord l'apprenant à l'association la plus divergente de l'aspect perfectif sur un procès statique dans «j'ai voulu». Le métalangage souligne le parti pris didactique en annonçant « une question de point de vue ", et requiert, il nous semble, un certain effort intellectuel en demandant d'imaginer par le prisme de la conscience d'un «locuteur» des faits comme des événements («Pierre a été charmant») ou comme des états ( ils allaient au cinéma hier soir »). 


\section{3. Limites de l'approche aspectuelle} choix énonciatif est formulée par Whatley (2011). Whatley a entrepris de tester l'approche aspectuelle de Dansereau (1987) en délivrant à des étudiants anglophones (États-Unis) dans leur deuxième semestre les explications standards du manuel constituées des listes d'emploi - accompagnées de la formulation de Dansereau (1987, cf. section 2.1. ci-dessus) pour un groupe, et les seules explication du manuel pour un autre groupe. Les données écrites sont recueillies sur un conte, où il faut choisir le temps approprié pour chaque verbe. La méthodologie ne comprenant ni verbalisation ou rappel stimulé, il est difficile de savoir quelles explications, le cas échéant, ont guidé les apprenants. Cependant, Whatley notait de façon similaire à Gündüz que l'approche aspectuelle aurait le défaut de laisser penser que le choix incombe à la seule volonté du sujet (2011: 654), tandis qu'à l'opposé les listes d'emploi du manuel induisent que ce choix est dicté par des indices contextuels.

\section{4. La perspective humaine incarnée dans l'énoncé}

D'après Gündüz (2005), les manuels de FLE généralistes (c'est-à-dire pour publics endolingues) ont abandonné l'approche aspectuelle représentée dans Libre Échange et qui devait amener à une prise de conscience de la vision distincte que les deux temps impliquent sur le procès. Par la suite, les manuels ont privilégié une approche « discursive ", centrée sur l'organisation du récit en un premier plan où l'on attend que les procès soient rapportés au passé composé et en un arrière-plan où ils seront à

Corela, $17-2$ | 2019 
l'imparfait. Gündüz (2005) remarque néanmoins que l'approche discursive ne permet pas de dépasser la convergence entre l'aspect lexical et l'aspect grammatical, c'est-àdire la tendance aux premiers stades qui fait associer passé composé et procès téliques et imparfait et procès atéliques (cf. section 1). Mais après avoir écarté l'option de l'approche aspectuelle, qui favorisait justement cette prise de conscience, voilà une observation qui peut sembler curieuse.

Une issue, que l'on pouvait attendre dans cette discussion mais qui n'a jamais été suggérée, serait de chercher à faire prendre conscience que les emplois prototypiques (passé composé sur procès ponctuels et imparfait sur procès statiques) ne sont pas moins des choix énonciatifs que dans le cas où ces associations divergent. L'exemple inévitable est l'imparfait "narratif », dit aussi "de perspective ", "stylistique», et même "pittoresque » employé sur des procès téliques : Une semaine plus tard, Charles épousait Emma. Du point de vue de l'acquisition, les associations divergentes ne seraient pas sans intérêt dans la démarche d'enseignement et devraient être incluses dans les données auxquelles les apprenants sont exposés (Shirai, 2016 : 21). Dans tous les cas, l'issue (manquée) de la discussion nous semble être que tous les verbes conjugués sont des choix énonciatifs d'une conscience avançant dans le monde qu'elle crée au fil des mots. Formellement, le système aspecto-temporel du français étend la distinction accompli / inaccompli sur tous les procès. En discours, cela signifie que ce choix est obligatoire. Certains choix sont «stylistiquement» visibles, alors qu'ils paraissent masqués dans les associations convergentes: Hier, j'étais à la plage; je discutais avec mes amis et Fabrice est arrivé avec une nouvelle petite amie. (exemple dans un manuel) Achard (2008) appelle « conventionnalisés » ces emplois où le choix énonciatif est masqué par l'usage. Habituel ou non, tout énoncé incarne une " perspective humaine » (Tomasello, 2003: 53), c'est-à-dire une façon de voir (la notion de construal en linguistique cognitive) commune au groupe linguistique mais intériorisée par le sujet qui l'a faite sienne. On envisagera, en conséquence, que la distribution des temps du passé dans les deux plans de l'organisation narrative est fonctionnelle dans le sens où elle guide, avec d'autre indices, l'interprétation du récit en remettant continuellement à jour cette perspective.

Alors que le CECRL était juste publié, Courtillon (2001) regrettait que la grammaire, cet " objet mythique », revenait dans les manuels de FLE présentée comme l'aboutissement de la leçon. L'esprit de l'approche communicative était (est encore) de soumettre la grammaire à ses intentions communicatives, et non l'inverse. Qu'en est-il à présent ? Toujours en nous bornant à la relation imparfait / passé composé dans le récit, la section suivante propose une analyse de manuels récents pour voir si la tendance décrite par Gündüz (2005) s'est confirmée (abandon d'une approche aspectuelle), ou si les concepteurs de manuels ont intégré les enjeux de choix énonciatif et d'organisation narrative.

\section{Analyse de manuels}

\section{1. Constitution du corpus}

À cette fin, on a choisi d'analyser les présentations grammaticales de la relation entre les deux temps dans le récit, et de procéder à un relevé de la première activité d'apprentissage où cette relation était mise à l'épreuve. Notre corpus comprend 17 
manuels généralistes, figurant tous dans le répertoire 2016 du CIEP (Centre International d'Études Pédagogiques) ${ }^{6}$. Pour obtenir des données homogènes, nous avons suivi la même procédure de recueil pour les 17 manuels : 1) copier la première occurrence dans la série des volumes où les deux temps sont présentés côte à côte, 2) décrire le type de la première activité la plus proche de cette présentation où le choix des temps est en jeu, 3) dans le cas de renvois à un précis grammatical pour la première étape ou un cahier d'exercices pour la deuxième, relever les contenus correspondants. Notons que certaines présentations incluent le présent et le plus-que-parfait, ce dont on n'a pas tenu compte par souci de clarté.

\section{2. Premier balayage de l'ensemble des manuels}

On s'intéresse d'abord aux présentations grammaticales. Le relevé des activités de pratique servira ultérieurement dans le cours de l'analyse. On observe tout d'abord que les présentations grammaticales peuvent être courtes :

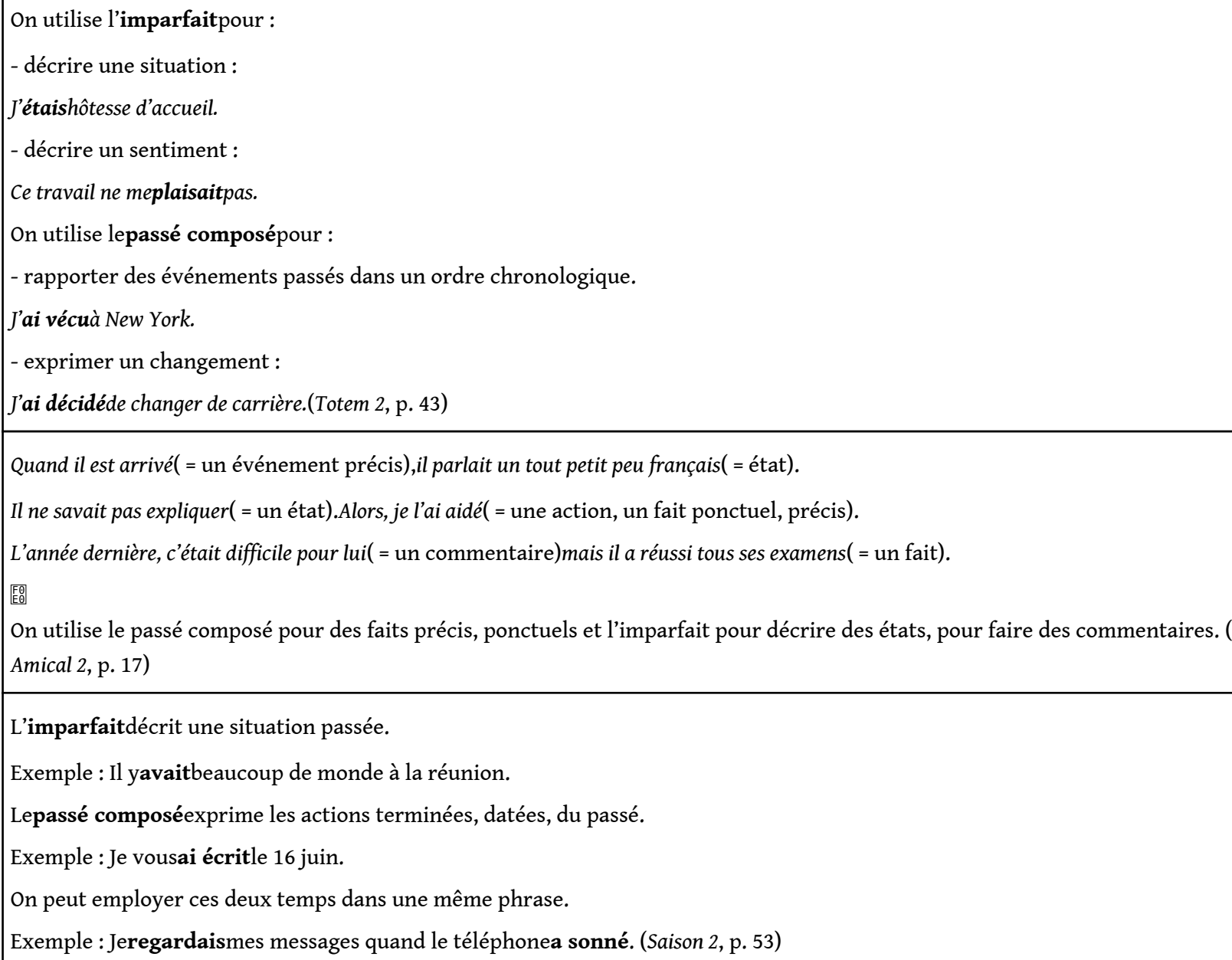

Tableau : exemples de présentations de la relation passé composé / imparfait dans trois manuels.

Si ces descriptions sont courtes, notons d'une part que les deux temps ont dans tous le cas été abordés séparément au préalable, et d'autre part que leur apprentissage 
continue au-delà de la page de la présentation grammaticale. Il y a très souvent un renvoi à un précis grammatical où la description peut être plus complète. Dans la leçon, une présentation succincte et simple est censée être adaptée à l'état transitoire de la langue apprise (interlangue). C'est là, d'ailleurs, le cœur du problème de la transposition didactique : si les termes des auteurs sont jugés par eux pertinents pour développer la maîtrise des temps verbaux, le métalangage pédagogique doit aussi nécessairement être valide devant les connaissances scientifiques, qui, elles sont des généralisations maximales. En cette matière, les trois exemples sont représentatifs : beaucoup de présentations grammaticales ne rendent pas évidente la distinction entre l'aspect grammatical et des propriétés portées par d'autres composantes de l'énoncé. En d'autres termes, les critiques de Dansereau (1987, cf. aussi section 2.1.) restent valables : terminologie devenue ambigüe en l'absence d'un point de référence (« actions terminées »), vague (" état », « description », « commentaire ») ou simpliste (« datées », avec un exemple comportant une date). Aussi, à l'exception de "j'ai vécu », un passé composé sur une activité, ces trois exemples sont également significatifs du fait que les concepteurs s'appuient largement sur le sémantisme des procès. Sur ce point, après l'observation des 17 manuels, on doit confirmer le constat de Gündüz (2005): l'approche purement aspectuelle de la relation imparfait / passé composé sous la forme de la présentation côte-à-côte du même procès sous les deux aspects inaccompli et accompli n'est tout simplement plus représentée. Cela signifie, concrètement, que les concepteurs de ces manuels n'ont pas jugé utile la seule opposition d'aspect pour la première présentation des deux temps côte à côte.

Un troisième constat d'ensemble est l'homogénéité des démarches dans tous les manuels, combinant repérage et observation des formes puis explicitation suivant les termes de l'encart grammatical. Ce n'est pas tout-à-fait une démarche de conceptualisation, dans la mesure où le travail réflexif des apprenants aboutit à la formulation prévue par le manuel. C'est ce que Puren (2001) avait déjà précisément décrit :

la conceptualisation grammaticale de chaque point de grammaire se fait de manière dite " active » dans le sens où ce sont les élèves qui l'effectuent eux-mêmes; mais dans cette conception classique de la « démarche inductive » en enseignement grammatical, l'enseignant garde en permanence comme référence et comme objectif la connaissance qu'il en a personnellement, et c'est pourquoi il se sent légitimé à exercer un contrôle étroit sur cette activité : sur la sélection des points abordés, sur le moment choisi pour le faire, sur le corpus et le métalangage utilisés, sur le processus de réflexion collective et enfin sur le résultat obtenu, à savoir l'énoncé des règles « standard » telles qu'elles apparaissent dans sa grammaire de référence ou dans le précis grammatical du manuel. (Puren, $2001: 138$ )

La conclusion de cette première vue d'ensemble est contrastée. Les critiques de Dansereau (1987) sont pour une bonne part toujours valables. Par ailleurs, le bannissement dans les encarts sur la relation entre le passé composé et l'imparfait d'exemples du même procès sous les deux aspects différents pose vraiment question. $Y$ a-t-il un tabou? Enfin, concernant l'homogénéité des démarches, le problème nous semble être dans le statut accordé par les acteurs (notamment les concepteurs et les éditeurs, mais aussi les enseignants, voire les apprenants) aux termes de la grammaire de référence et à la fabrication du consensus sur les critères qui l'établissent comme 
telle - mais cette question dépasse le cadre de cette étude -, et non dans la phase heuristique d'observation, qui est reconnue utile.

\section{3. Sélection d'un manuel intégrant choix énonciatif et organisation narrative}

Dans un premier temps, on avait noté les termes récurrents tels que pour le passé composé "actions précises, ordre chronologique, fait "avancer" le récit, etc. ", en espérant pouvoir dégager les traits les plus nombreux. Mais la variété des termes s'est avérée problématique. Surtout, chez le même éditeur, on a noté que des formulations sont parfois reprises à peine modifiées ou même à l'identique d'un titre à l'autre. Dans cette situation, la part quantitative de l'analyse n'avait plus d'intérêt évident. On a opté pour l'analyse qualitative du manuel qui aurait le mieux réalisé l'intégration du choix énonciatif dans l'organisation narrative, c'est-à-dire, qui traiterait, de notre point de vue, de façon la plus appropriée le problème des « emplois conventionnalisés ».

En observant les termes collectés en premier balayage, et en écho à l'approche fonctionnelle-notionnelle (cf. section 2.1.), on a entrepris de classer les manuels selon que ces termes se rapportent à deux catégories de sens. Dans la première, il nous semble que l'on peut inférer des présentations grammaticales la présence d'un observateur. En d'autres termes, ces présentations peuvent suggérer à l'apprenant que les temps verbaux causent un effet perceptif dont il est à l'origine comme énonciateur. Les métaphores spatiales tels que «intérieur / extérieur, arrière-plan / premier plan, déroulement" ainsi que les termes temporels tels que "actions terminées, limitées dans le temps, fait ponctuel, etc. " relèvent de cette première catégorie. La seconde ressemble les notions présentées comme indépendantes d'un observateur. Les termes tels que «actions datées, situation / action, décor, circonstances, commentaire » s'y rapportent.

Pour contourner le problème des reprises éditoriales d'un titre à l'autre, on a sélectionné un manuel dans chacune des maisons d'édition représentées dans notre corpus dont les présentations grammaticales étaient les plus riches en nombre d'éléments se rapportant à ces deux catégories ${ }^{7}$. On a ensuite comparé ces manuels entre eux suivant la même procédure. En d'autre termes, c'est dans le manuel choisi au terme de cette sélection, Version Originale 2, que l'apprenant est susceptible d'être exposés à des exemples présentés comme des choix énonciatifs dont il est responsable pour l'intelligibilité du récit, et non pas seulement comme des modèles à suivre en considérant des indices donnés pour indépendants de sa présence.

\section{3. Analyse de la présentation grammaticale dans Version Originale 2 (2010)}

L'encart grammatical du manuel Version Originale 2 est reproduit ci-dessous (tableau 4). Son contenu sera glosé, ainsi que l'activité de choix dans la section suivante, en recourant parfois à des comparaisons avec d'autres manuels.

PARLER DE FAITS PASSÉS

LE PASSÉ COMPOSÉ / L'IMPARFAIT 
Dans le récit, le passé composé présente les actions comme des faits terminés, observés « depuis l'extérieur ». C'et le temps qui fait « avance

Martineest arrivéechez ses parents vers trois heures, elleest entréepar la porte principale eta essayéd'allumer la lumière. Ellea enten

L'imparfait présente les actions comme des circonstances observées« depuis l'intérieur», comme des circonstances qui entourent les autres

Martine est arrivée chez ses parents vers trois heures, ilfaisaitfroid et la rueétaitsilencieuse. Elle est entrée par la porte principale, qui pas fermée comme d'habitude et elle a essayé d'allumer la lumière. Elle a entendu des bruits quivenaientdu premier étage, celaressemb

À l'oral et souvent à l'écrit, on utilise aussi le présent pour raconter des événements passés. Cela rapproche le récit du temps actuel.

Un jour, j'étais seul à la maison. Tout à coup, j'entendsun bruit étrange quivientde la cuisine.

\section{SE PASSER}

Pour demander des précisions sur un événement, on peut utiliser la questionqu'est-ce qui s'est passé ?

Qu'est-ce que ce qui s'est passé?

-Je ne sais pas, maman, je faisais mes devoirs. (Accompagné d'une illustration, où l'on voit une fenêtre cassée, et le petit garçon tenant

Tableau : présentation de la relation imparfait / passé composé dans le manuel Version Originale 2 (p. 75).

Il s'agit parmi les 17 manuels du corpus de la seule explication basée sur la relation entre un observateur implicite et les faits perçus («faits terminés, observés depuis l'extérieur ", " circonstances observées depuis l'intérieur»). Si l'on veut identifier des descriptions théoriques dont on peut rapprocher ces termes, la "fenêtre de monstration » (Victorri, 1999) ou "l'intervalle de référence » (Gosselin, 2005) - notion construite sur la précédente métaphore de la fenêtre - viennent à l'esprit. Gosselin (2005 : 36) distingue accompli du présent (la base morphologique du passé composé à valeur de perfectif passé) et inaccompli dans l'imparfait par l'endroit où opère l'intervalle, respectivement à droite de la borne droite du procès, donc à l'extérieur du procès, pour le passé composé, mais à l'intérieur des bornes du procès - vision sécante - pour l'imparfait.

Cette dichotomie " depuis l'extérieur » / " depuis l'intérieur » est également justifiable d'une analyse en terme de référence temporelle. La première alternative - le passé composé - correspond au fait que l'on voit le passé depuis le présent, en établissant une distance dans le temps (à partir de la deixis) spatialisée sous un rapport d'extériorité. $\mathrm{Au}$ contraire, « depuis l'intérieur » renvoie à la valeur anaphorique de l'imparfait, qui fonctionne en relation dépendante avec un marqueur temporel de la situation passée (dans l'exemple du manuel, "vers trois heures»). Mais ici la notion de point de référence n'est pas explicite. Dansereau (1987) estime que cela exigerait de traquer ce point de référence de situation en situation, ce qui introduirait un coût inutile pour 
l'apprentissage. Au contraire, Labeau (2002) y est favorable, tout comme Gündüz (2005), comme on l'a vu.

La structure même des exemples de l'encart dédouble l'opposition "extérieur " / « intérieur». Les exemples constituent un même récit, dont d'abord seule la trame narrative de premier plan est rendue, puis à laquelle s'ajoutent les « circonstances » des actions. L'effet d'ajout pour l'apprenant est aussi visuel :

"Martine est arrivée chez ses parents vers trois heures"

"Martine est arrivée chez ses parents vers trois heures, il faisait froid et la rue était silencieuse »

Surtout, le choix est de montrer l'arrière-plan dans un second temps, à partir, d'abord, de la première focalisation sur la trame. Avec l'imparfait, on s'introduit dans le monde ouvert par les mots (dans la "scène verbale ») pour fixer le fond duquel les actions se détachent. Les apprenants sont implicitement exposés à l'idée que l'arrière-plan précède les actions qu'il explique : «Elle est entrée par la porte principale, qui n'était pas fermée comme d'habitude ». Une autre manuel exploite aussi l'idée que le passé composé ouvre le monde du récit : «Le passé composé introduit l'action (comme dans un film) » (Édito A2, p. 142). Néanmoins, du fait que l'arrière-plan est indépendant de la perception des événements de la trame (Reinhart, 1984), le choix de commencer par l'imparfait se justifierait aussi à notre sens.

La présentation grammaticale de ce manuel fonctionne également sur le rôle respectif des temps dans l'organisation du récit, même si les termes "premier plan" et "arrière-plan" n'apparaissent pas ${ }^{8}$. L'option prise, unique dans le corpus ${ }^{9}$, est d'exploiter la perception du rythme du récit : le passé composé « fait avancer » le récit dans la succession des actions, mais l'imparfait "arrête» le récit. Pour le passé composé par exemple, les formulations dans les autres manuels se bornent, le cas échéant, à parler d'un temps qui présente les " événements qui se succèdent " (À propos A2, p. 116), les « événements passés dans un ordre chronologique " (Totem 2, p. 43). Le potentiel opératoire de la distinction entre "faire avancer» / "arrêter le récit» mériterait d'être testée, mais les concepteurs établissent une symétrie fonctionnelle, là où les autres manuels (à l'exception de la distinction des plans dans Alors 2, voir note 8) ne définissent pas le rôle parallèle de l'imparfait.

Un autre avantage est que cette conceptualisation de l'imparfait (guidée - comme tous les autres manuels, la leçon a commencé par une phase heuristique) est qu'elle ouvre la voie à un dépassement de l'association convergente entre procès atélique et aspect grammatical imperfectif (voir tableau 1). Cette présentation grammaticale arme les apprenants pour apprécier les imparfaits narratifs, qui sont par exemple suremployés dans les résumés de matchs de football ou de rugby.

\section{4. Analyse de l'activité de choix du temps}

Sur l'ensemble du corpus, on avait aussi relevé la première activité après la présentation grammaticale dans la leçon qui exerçait au choix entre les deux temps. Avec les réserves que l'on a indiquées pour une exploitation quantitative des données du corpus, on observe que le type d'activité qui domine est la transformation de texte. Sur 17 manuels, 8 proposent un texte avec les infinitifs à conjuguer, 5 un texte au présent à mettre au passé, 2 des phrases avec les infinitifs à conjuguer ${ }^{10}$. 
Pour une analyse qualitative, on s'intéressera à l'activité du même manuel, Version Originale 2, qui comme pour la présentation grammaticale, se distingue par sa créativité (tableau 5):

\section{LE MYSTÈRE DE JULIETTE K}

A. Il y a quelques jours, Juliette K. est allée passer un week-end dans la maison de campagne de ses parents. Écoutez l'enregistrement et pre

B. Écrivez le récit de ce qui s'est passé. Attention : vous devez utiliser au moins 10 des expressions suivantes.

maison de campagne, voiture, courir, lumière, forêt, allumettes, verre, yeux verts, cuisine, escalier, chat, piano, bruit, assiette, porte, casser

Tableau : activité pour l'alternance imparfait/passé composé dans le récit (Version Originale 2, p. 76).

44 Ce que les apprenants ont écouté est en réalité une bande-son, c'est-à-dire des bruits, et de brefs motifs musicaux d'ambiance: pluie battante, grondements de tonnerre, arrivée d'une voiture, porte, aboiements, pas, tintement d'un trousseau de clés, grincement d'une porte, bruit de la pluie battante estompé en arrière-plan, allumette qu'on craque, pas, porte grinçante, accords dissonants au piano, pas dans un escalier, verre cassée, porte, accords dissonants au piano, miaulement, hurlement, pas précipités, porte, pluie battante, accords dissonants, tonnerre, miaulement.

45 À la fin de la première écoute, la forme qui apparaît - le guide pédagogique suggère de faire fermer les yeux - est l'intervalle de temps relativement silencieux pendant lequel le bruit de la pluie battante est juste deviné en arrière-plan, c'est-à-dire quand l'héroïne est dans la maison. Un passé composé résomptif s'impose : elle est entrée dans la maison et en est ressortie. Est-ce que la présentation grammaticale permet de produire cette première vue d'ensemble? Les concepteurs de l'activité ont pu vouloir exploiter l'opposition "depuis l'intérieur / depuis l'extérieur». La séquence sonore s'ouvre et se clôt sur le bruit de la pluie, qui n'a pas cessé. La visite de la maison semble s'en détacher comme une forme, observable, comme la maison, « depuis l'extérieur ».

46 Un autre aspect de l'activité est, en comparaison des autres manuels, la tentative de faire vivre une expérience sensorielle directe, mais non dénuée d'humour: les apprenants peuvent reconnaitre les codes du film de suspense et en jouer dans leur production écrite. Sur le seul plan du maniement des temps verbaux, l'activité repose sur les associations convergentes (l'imperfectif sur "pleuvoir, être la nuit», etc., le perfectif sur « arriver, ouvrir, fermer, etc. ». C'est la cas des activités de transformation de texte dans les autres manuels. Celles-ci peuvent avoir un intérêt, en particulier si elles sont accompagnées de verbalisation entre et avec les apprenants pour qu'ils justifient leurs choix. Mais ce type d'activité a un inconvénient majeur : les apprenants seront tentés de chercher des indices dans le cotexte, tels que les marqueurs de durée, moment, fréquence mais aussi le sémantisme des procès. Ce n'est pas qu'il ne faille pas faire s'exercer à la synthèse de ces composantes. C'est que la médiation d'un premier texte prive de la liberté de construire cette synthèse.

Un exemple est l'association convergente sur "enflammer» ou mieux, pour le phonosymbolisme, «craquer une allumette ». Quand on identifie le son de l'allumette que l'on enflamme en écoutant l'enregistrement, elle est déjà enflammée. Ce patron où perception et verbalisation se succèdent immédiatement est un principe d'acquisition observé pour la morphologie verbale de l'accompli. Il a été noté en effet que dans l'acquisition de sa langue, l'association entre la morphologie verbale marquant 
l'accompli, le temps verbal évoquant le passé et les procès ponctuels est quasiment systématique. Dans le commentaire d'un changement soudain intervenu dans l'environnement, l'enfant entend des marques de l'accompli associés au passé, parce que ce changement aura été trop bref pour qu'on l'ait commenté dans son cours (Shirai \& Andersen, $1994: 148$ et sq. ; Shirai, 2009 : 176). Le français, comme d'autres langues romanes, a développé l'accompli sur une structure possessive pour retenir dans le présent (cela indéfiniment en français) les faits juste perçus mais irrévocablement passés. On y reviendra.

Après que les actions ont été décrites suivant la logique fonctionnelle de l'accompli, et pour installer la dépendance des actions de la trame à l'égard du fond, l'enseignant peut faire "arrêter » le récit et orienter "depuis l'intérieur » vers les circonstances de l'action (en fonction des feedbacks nécessaires) : pourquoi est-ce qu'elle a craqué une allumette?

\section{Proposition de matériel et éléments de mise en œuvre}

\section{1. Principes directeurs}

Le matériel dont des éléments sont décrits ci-dessous est actuellement en phase de conception et de test, il est donc susceptible d'être modifié à l'avenir. Il est prévu pour des étudiants de niveau initial qui n'ont pas encore abordé l'alternance des temps du passé. L'objectif poursuivi est d'aider à l'acquisition des effets perceptifs produits dans les énoncés avec l'imparfait et le passé composé. Ces effets sont intrinsèquement mis en jeu dans l'organisation narrative du récit pour guider l'interprétation de l'interlocuteur / du lecteur, et l'orienter (en s'orientant soi-même) dans le monde du récit en train d'être raconté. Le matériel exploite l'idée que l'emploi des temps tient à jour la perspective prise par le sujet, laquelle est une pratique collective intériorisée, c'est-àdire un choix énonciatif reconnu (voir précédemment section 2.4.).

Toutefois, à la différence de l'exemple analysé dans la section précédente, le matériel exploite également la différence morphosyntaxique entre l'imparfait (temps simple) et passé composé (temps composé). Du point de vue de l'expérience de parole que les énoncés avec l'un ou l'autre des temps imposent, la nature de l'état dans lequel le sujet énonciateur se met est différente : percevant dans un présent passé (ou imaginaire) dans le cas de l'imparfait, "possesseur » actuel d'un fait accompli dans le passé (si c'est un récit) avec le passé composé. Le point de vue théorique emprunte au cadre de la linguistique énactive (Bottineau, 2010) et à des positions adjacentes sur la prosodie (Auchlin, 2017) et la syntaxe (Poirier, 2017). Notamment, la syntaxe et la prosodie coordonnent le processus actif de l'échange vocal. L'ordre syntaxique, ou plus précisément la prosodie particulière qui se modèle sur cet ordre, est inévitablement le même pour les participants à l'échange, lesquels sont comme condamnés à suivre le débit (Auchlin, 2017). Le sens réalisé dans l'énoncé sera comparable, du fait d'un code partagé, mais particulier aux individus. La perspective énactive récuse la vision d'un sens prédonné - l'accompli / l'inaccompli - encodé dans des formes acoustiques - $[\varepsilon$ vy] / [vwaje]. On peut dire que plutôt que d'opérer la mise en mots d'idées stockées en mémoire, le sujet lui-même se met en mots. Il doit se plier à leurs formes, il respecte les règles d'agencement des unités linguistiques et les façons de prononcer pour être 
intelligible. La linguistique énactive suggère que ces contraintes soutiennent des opérations d'élaboration progressive du sens linguistique (voir dans Poirier (2017) les principes proposés d'une "chronosignifiance»). À partir de ces positions, on s'intéressera à l'expérience induite dans l'énonciation de la relation syntaxique sujet verbe - complément dans le cas d'énoncés au passé composé et à l'imparfait. À notre sens, la différence morphosyntaxique contribue à la distinction aspectuelle des deux temps. En particulier, la présence du participe passé dans une construction possessive pour le passé composé introduit une marque asymétrique productive. La proposition de ce matériel et ses éléments de mise en œuvre reposent aussi sur l'hypothèse que le point de vue adopté est bénéfique pour l'apprentissage.

\section{2. Caractéristiques du matériel et éléments de mise en œuvre}

51 À l'instar du manuel Libre Échange (cf. section 2.2.), on opte pour la présentation du même procès sous les deux aspects différents. L'avantage est de permettre la prise de conscience (progressive) de la valeur intrinsèque de l'aspect grammatical. La présentation du matériel aux apprenants se fait lors de son utilisation au moment de l'activité de pratique, dont le support est un récit d'images, reproduit ci-dessous (illustration 1). Le récit est en première personne, l'apprenant rapporte l'expérience à un(e) ami(e) :

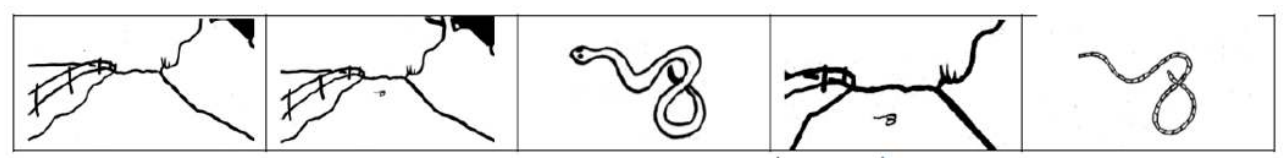

Illustration : support de la première activité.

Trois types de récit peuvent être attendus, comportant les verbes au présent, à l'imparfait et au passé composé ou à ces trois temps (tableau 6). Pour les besoins de la démonstration, on s'intéressera au second exemple, mais le présent de narration est fréquemment employé (cf. Weinrich, 1973 : 302). Notons aussi, suivant les études rapportées par Véronique et al. (2009: 206), que les formes du passé sont suremployées dans la classe pendant la période où elles sont étudiées, pour des emplois où les locuteurs natifs raconteront souvent au présent ${ }^{11}$.

\begin{tabular}{|c|c|c|}
\hline Présent & Imparfait et passé composé & Présent, imparfait et passé \\
\hline $\begin{array}{l}\text { Tu sais quoi ? Ce week-end, jevais } \\
\text { me promener dans la campagne, comme d'habitude. Je } \\
\text { marchesur un chemin, et tout à coup jevois } \\
\text { quelque chose. J'aipeur. Jepenseque c'est } \\
\text { un serpent! Jeme rapprocheet en fait c'estune corde! }\end{array}$ & $\begin{array}{l}\text { Tu sais quoi ? Ce week-end, jesuis allé } \\
\text { me promener dans la campagne, comme d'habitude. Je } \\
\text { marchaissur un chemin, et tout à coup j'ai vu } \\
\text { quelque chose. J'ai eupeur. J'ai penséque c'était } \\
\text { un serpent! Jeme suis rapprochéet en fait c'était } \\
\text { une corde! }\end{array}$ & $\begin{array}{l}\text { Tu sais quoi ? Ce week-end, } \\
\text { me promener dans la camp } \\
\text { marchesur un chemin, et to } \\
\text { quelque chose. J'ai eupeur. } \\
\text { un serpent! Jeme rapproch } \\
\text { une corde! }\end{array}$ \\
\hline
\end{tabular}

Tableau : exemples possibles de productions pour le récit d'images.

53 Les visuels du matériel prennent comme exemples des verbes des activités. L'illustration 2 ci-dessous présentent l'alternance «je marchais » / «j'ai marché ». Le 
cas de «je voyais » / «j'ai vu» sera abordé plus bas. À partir de deux extraits, on passera en revue certaines des caractéristiques du matériel concernant ses objets d'apprentissage, l'imparfait, le passé composé, le plus-que-parfait, les types de procès et l'organisation narrative en indiquant également des éléments de mise en œuvre, avant de conclure l'ensemble de cet article.

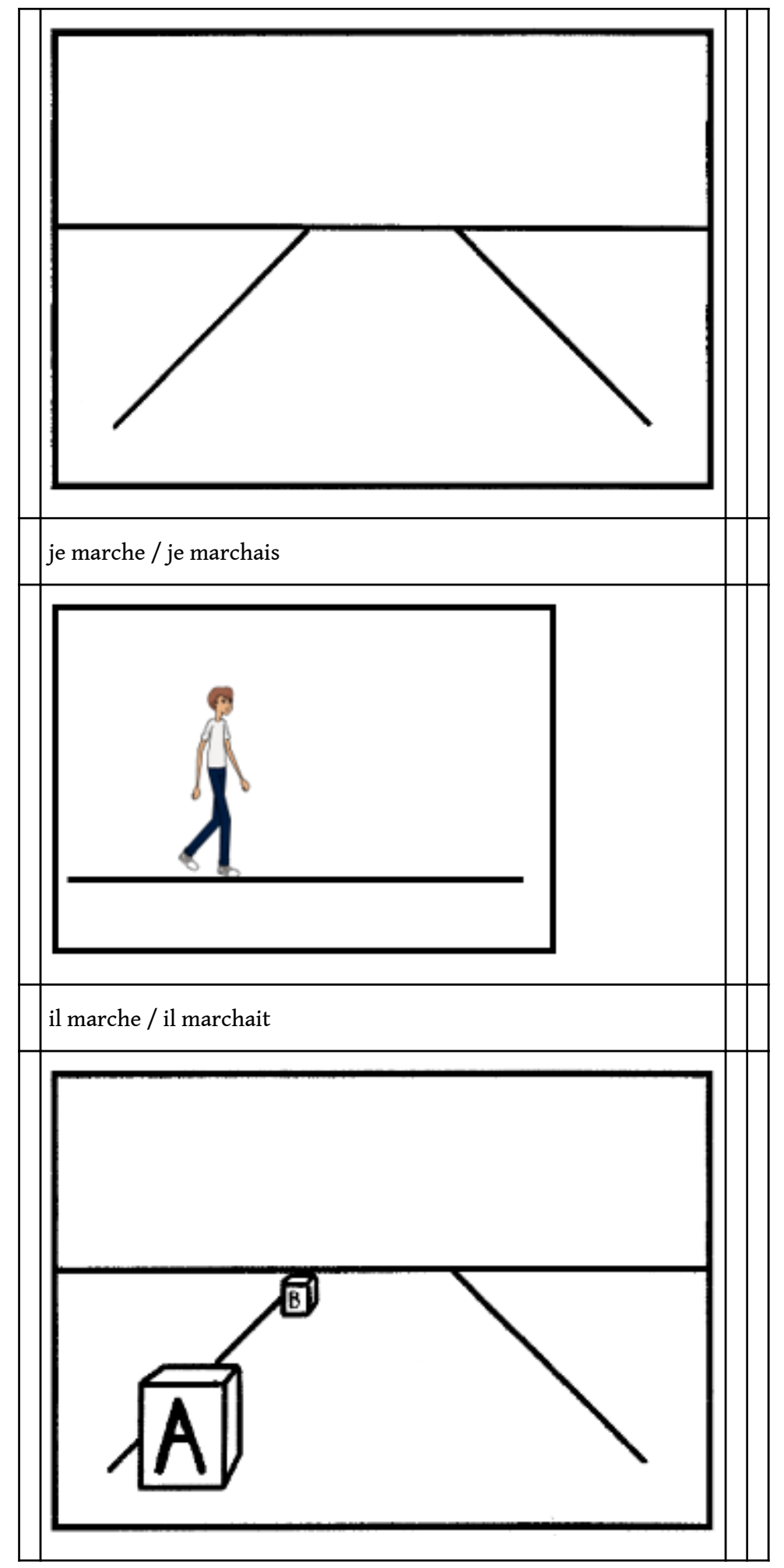




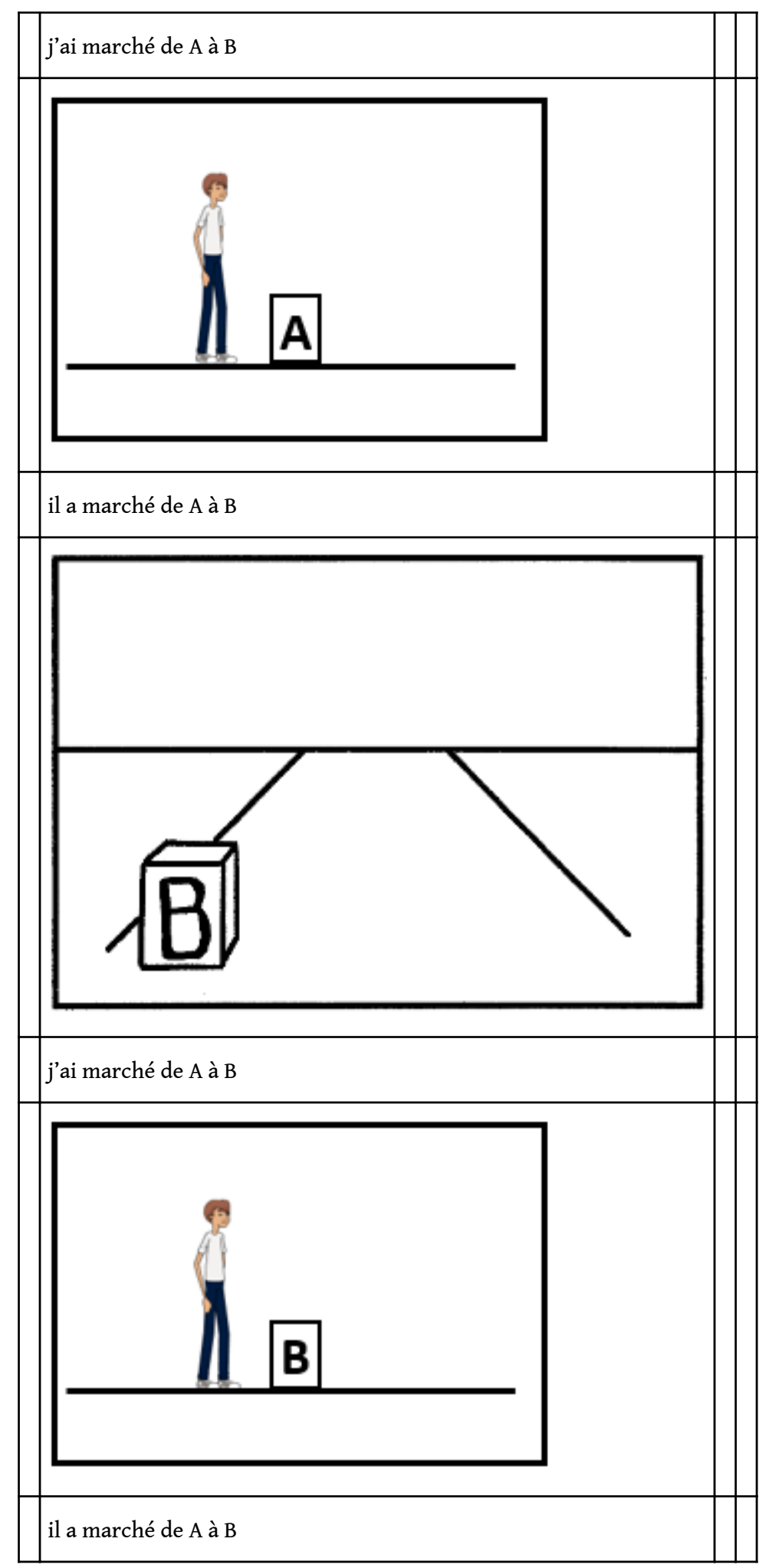




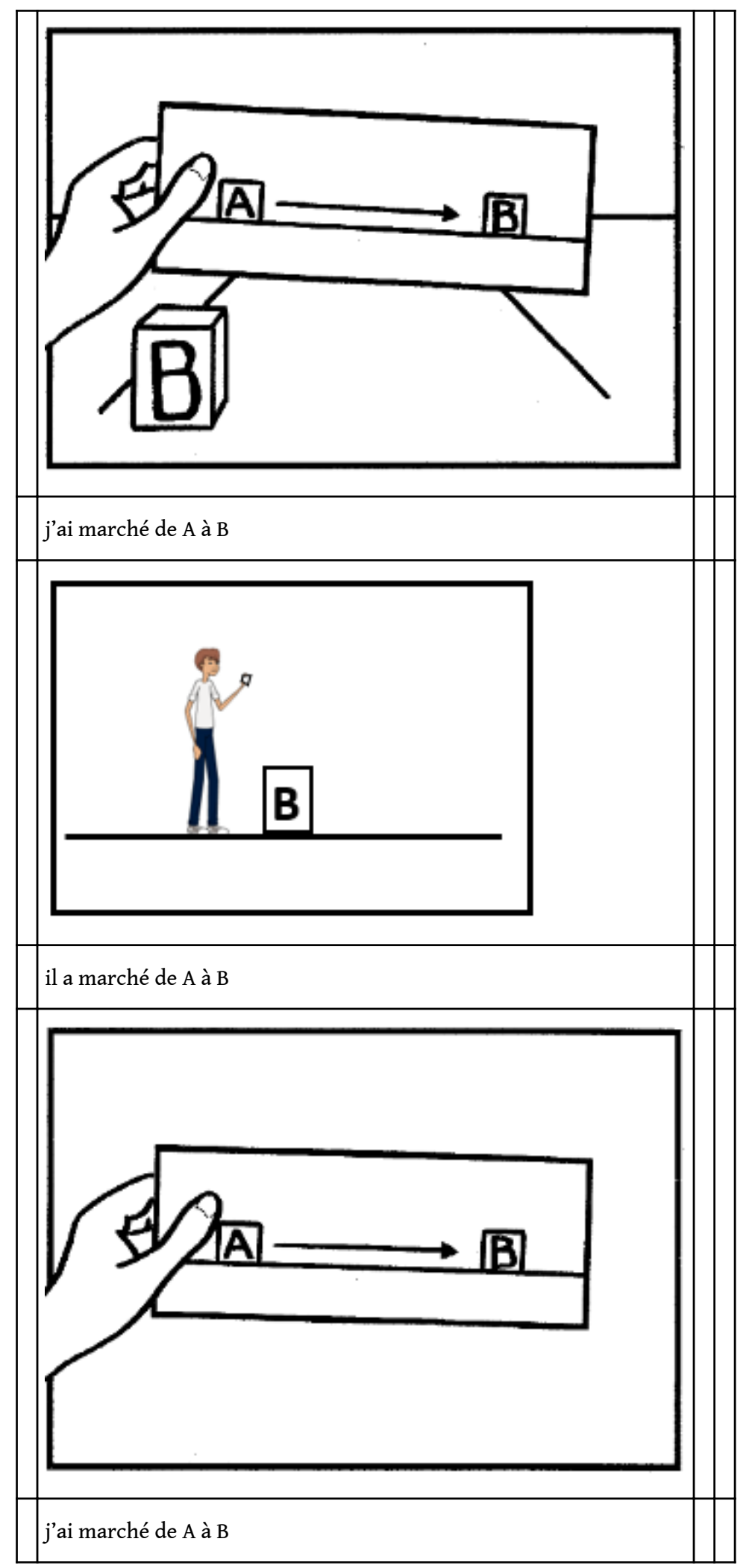




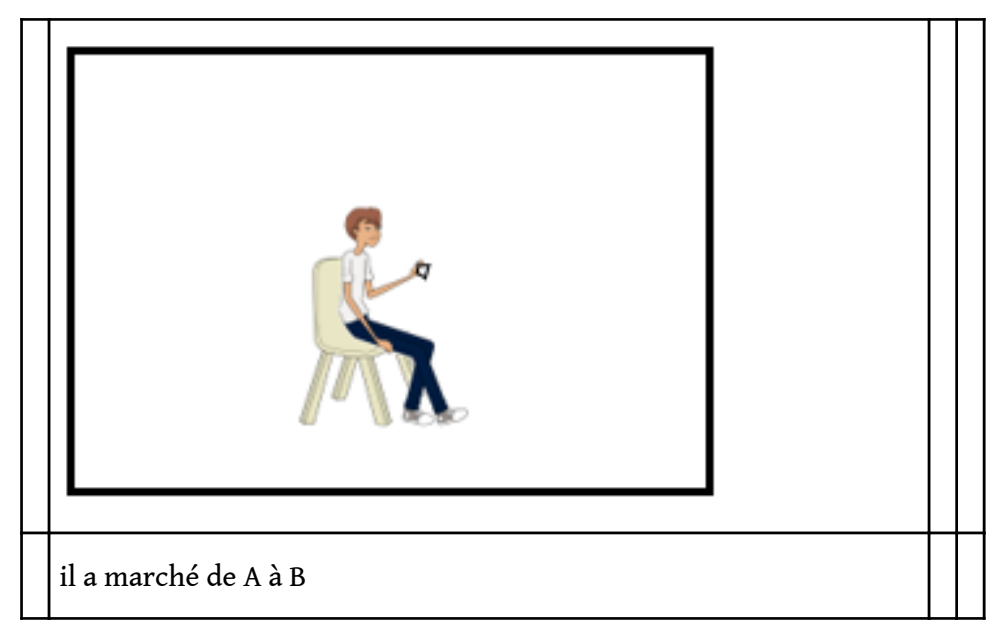

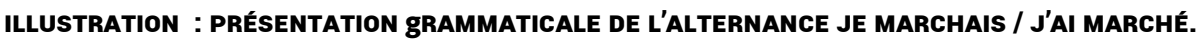
l'apprenant l'identité des dynamiques perceptuelles du présent et de l'imparfait (Bottineau, 2014). L'espace ouvert devant le sujet percevant, et en comparaison avec l'image des bornes pour le passé composé plus bas, est censé aider à clarifier l'erreur commune d'associer imparfait à durée. Cette erreur relève d'une simplification pour dire que ce qui est perçu perdure sans changer ${ }^{12}$. Lors de la présentation du matériel si nécessaire, le professeur peut étendre les données linguistiques à d'autre procès, pour instiguer le sens de l'imparfait à partir du présent : l'imparfait est un présent qui n'est ni ici ni maintenant. Ainsi, «"je marchais" est comme “je marche ”, mais pas ici ni maintenant » ou « "je marche, ici et maintenant”, “ je marchais, ailleurs et à un autre moment" " nous paraissent être des paraphrases pédagogiques acceptables. Elles peuvent être déclinées sur d'autres verbes, afin d'exposer les apprenants à la valeur aspectuelle (et modale) de l'imparfait, et pour enseigner l'expérience subjective suscitée par l'énonciation d'un imparfait ${ }^{13}$.

- Le passé composé

Les visuels pour le passé composé narrent une séquence d'actions qui aboutit aux énoncés de l'accompli du présent puis de l'accompli dans le passé, les deux ayant la même forme « j'ai marché de A à B ». À notre sens, l'origine de la syntaxe complexe du passé composé est à chercher dans notre réalité cognitive. Sa composition est un moyen pour, assez littéralement, reprendre la main sur le passé, pour retenir le « moment précédent » de l'environnement percevable car

58 [à] chaque moment qui vient, le moment précédent subit une modification : je le tiens encore en main, il est encore là, et cependant il sombre déjà , il descend au-dessous de la ligne des présents ; pour le garder, il faut que je tende la main à travers une mince couche de temps. (Merleau-Ponty, [1945] $2016: 478$ )

Ma main tenant l'accompli : c'est l'expérience que fait vivre la structure possessive du passé composé (avec l'auxiliaire « avoir ») ${ }^{14}$. Même grammaticalisé en perfectif passé, la dernière case du visuel, il reste un temps du corps parlant ici et maintenant mais qui réalise ce geste. Dans cette perspective, on suggère que l'on aurait intérêt à présenter aux apprenants les énoncés au passé composé à partir de l'expérience qu'elle cause 
chez le sujet. Pour caractériser cette expérience, on peut rappeler l'origine de la construction.

L'auxiliaire habere dans la périphrase habere + participe passé s'est d'abord imposé sur mihi est comme verbe de possession : mihi est pecunia cède devant habeo pecuniam. Dans cette évolution, le sujet grammatical n'est donc plus pecunia, mais ego, qui est « le siège d'un état " (Benveniste, 1996 : 197). Dans la périphrase habere + participe passé qui dominera en latin parlé, « l'auteur du procès est désigné comme possesseur du résultat, qui lui est acquis.» (Benveniste, 1974: 129, italique dans l'original) De même en français, le passé composé fait du sujet un possesseur de l'accompli. Notre visuel essaie de représenter cet état. En outre, les éléments dessinés reproduisent le sens opéré dans l'ordre syntaxique : la main pour « j'", le maintien pour « ai » et l'encart pour « marché de $\mathrm{A}$ à $\mathrm{B}$ ». Le participe passé, une forme nominale du verbe, entre comme objet dans des constructions connues des apprenants initiaux: «j'ai de l'argent / je n'ai pas de l'argent ». On estime que l'enseignant doit exposer les apprenants à des énoncés au passé composé en se faisant vivre le même état d'esprit que dans des énoncés avec "avoir", pour évoquer la possession du résultat de l'action comme on évoque la possession d'un objet. La gestuelle peut contribuer à l'expérience de la construction du sens, en s'improvisant autour du thème de la main «tenant » le résultat. Une gestuelle appropriée peut aider à vivre l'expérience distincte de celle de l'imparfait.

61 - Le plus-que-parfait

62 Le plus-que-parfait s'aborderait comme l'imparfait, en exploitant l'identité des dynamiques perceptuelles entre présent et imparfait, mais à partir de l'accompli du présent. Pour cette raison, on estime que, suivant la logique suivie dans la conception de ce matériel, il serait plus facile d'utiliser des verbes téliques, qui convergent facilement avec l'accompli : “ “j'avais fini” est comme "j'ai fini ”, mais pas ici ni maintenant" ", etc.

\section{- Les types de procès}

64 Notre matériel comprend des exemples d'emplois à l'imparfait et au passé composé sur trois procès, « marcher ", " voir quelque chose sur le chemin » et « écrire son rapport ». Ces verbes sont extraits du lexique des activités. Les trois verbes consistent en des procès atélique, ponctuel ("voir» dans le sens d'«apercevoir») et télique. On ne présentera pas les visuels pour le dernier. L'association divergente sur les procès statiques est abordée à partir de l'exemple sur "marcher»: ces deux types fonctionnent avec le marqueur " pendant » (Renaud, 2002) que nous avons illustré par des bornes.

Mais de toute évidence, l'association divergente est un défi pour l'enseignant : «J'ai eu peur. J'ai pensé que c'était un serpent!» ou «J'avais peur. Je pensais que c'était un serpent!». La préférence va à la première alternative du fait que l'ancrage de la structure possessive dans le présent est pragmatique : «Regarde! Ces expériences sont extérieures à moi, je les tiens et elles ne me font plus grand-chose !» Pour cette raison, "J'avais peur " paraît inacceptable. Quant à «était », l'identification (erronée) du serpent engage le présent du sujet percevant in media res. "Je pensais » est possible si l'on rapporte l'action de penser à l'identification, mais "J'ai pensé » sera utilisé si on ferme cette action à l'extérieur de soi pour l'exhiber à notre interlocuteur.

66 - L'organisation narrative 

extension métaphorique des principes de la perception visuelle. Essentiellement, deux idées nous paraissent devoir être soulignées pour l'apprentissage de la maitrise des temps du passé dans le récit. D'abord, les actions de la trame narrative dépendent de l'arrière-plan, de même que les figures sont perçues par rapport à un fond dont elles se détachent. En second lieu, la trame de premier plan a des propriétés perceptuelles particulières: 1) la succession des actions de premier plan, l'ordre chronologique qu'elles suivent (ce sont des termes souvent rencontrés dans les présentations grammaticales du corpus) établissent une continuité, qui, selon la «loi de bonne continuation » est prioritairement perçue comme une figure, 2) les procès ponctuels se trouveront plus facilement au premier plan, de même que les zones étroites sont plus aisément perçues comme figures, 3) le parallèle vaut également entre l'accompli et les formes closes (Reinhart, 1984 : 801-805).

Dans ce rapprochement, cette auteure suggère que l'organisation narrative en deux plans est une nécessité pour l'intelligibilité du récit, et cela indépendamment de la langue. Les visuels pour "voir» (des éléments sont présentés ci-dessous dans l'illustration 3) pourraient permettre un rapprochement implicite des deux modalités, visuelle et linguistique, de la perception des actions sur ces deux plans.

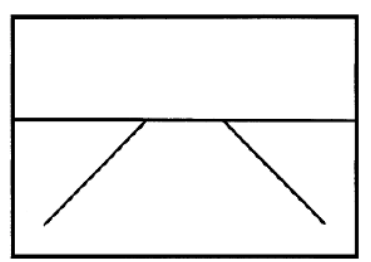

je vois / je voyais le ciel je vois / je voyais le chemin

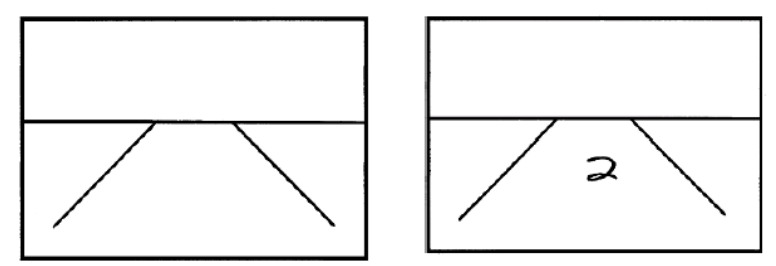

j'ai vu quelque chose sur le chemin

Illustration : éléments du matériel pour l'alternance je voyais / j'ai vu.

Pour le passé composé, il s'agit des deux premiers visuels (on ne reproduit pas la «tenue » du participe passé dans la main : le principe exploitant la structure possessive est le même). Dans le premier de ces visuels, l'objet sur le chemin n'est pas vu par moi, mais il l'est peut-être pour un autre observateur. C'est mon action, la marche, dans le monde que je décris («je marchais »), qui rend possible le fait de voir : l'arrière-plan est le plan des causes des actions perçues au premier. De même, la perception d'une figure étroite (l'objet) sur un fond (le chemin) dont elle se détache étant facilement associée à la trame de premier plan, comme l'argumente Reinhart (1984), ces deux premières cases devraient aider l'apprenant à développer implicitement la compréhension du rôle du passé composé dans l'organisation narrative. Enfin, si « je voyais » est l'état de voir une entité, "le chemin ", déjà là avec moi (valeur anaphorique de l'imparfait), le fait que cet état perdure encore quand j'aperçois l'objet puis après que je l'ai aperçu doit contribuer à l'association de la forme en $[\varepsilon]$ avec l'arrière-plan.

\section{Conclusion}

L'apprentissage de langue étrangère est un processus humain de multiples dimensions, lequel varie aussi en fonction du domaine linguistique enseigné. L'acquisition de la distinction accompli / inaccompli dans le récit au passé par des publics non natifs 
dépendra partiellement des langues de départ. L’acquisition sera rendue difficile, $a$ priori du moins, si le français est typologiquement éloigné. Le cas échéant, le métalangage introduit en scolarisation pour la langue première, puis pour des langues étrangères avant l'exposition au français entre de surcroît en ligne de compte. Ces questions, nombreuses et essentielles, n'ont pas été abordées. Pour l'expérimentation du matériel en contexte exolingue, prévue ultérieurement, les enjeux d'influence translinguistique, du rôle métalinguistique de la langue de départ, du métalangage antérieurement connu (pour l'anglais généralement) devraient être pris en compte.

71 La présente étude a d'abord consisté à rapporter les termes d'un débat sur l'approche d'enseignement de l'emploi du passé composé et de l'imparfait dans le récit, en particulier en ce qu'il a concerné la conception de matériel pédagogique. C'est souvent la critique du matériel existant et la remise en cause des démarches d'enseignement qui l'ont alimenté (cf. Dansereau, 1987). Les auteures du manuel Libre Échange (2001), dont on analysé un extrait, avaient choisi une approche "aspectuelle» (Gundüz, 2005). L'apprenant était exposé à des données où les notions de résultat ou d'état étaient significativement mises en jeu: «je n'ai pas pu» vs. «je ne pouvais pas». Mais notre analyse de 17 manuels généralistes publiés entre 2006 et 2016 ( 5 maisons d'édition représentées) confirme le constat de Gündüz (2005) : l'approche de Libre Échange a été, globalement, délaissée. Dans notre corpus, il n'y a pas un seul exemple d'un même procès présenté sous les deux aspects différents. Cela tiendrait, s'est-on demandé, du tabou. On a ensuite analysé plus en détail un manuel de notre corpus, Version Originale 2, où les formulations grammaticales prennent tout de même en compte la possibilité d'un choix de perspective sur le procès. L'activité de pratique, une immersion dans un récit sonore à écrire au passé, semble pouvoir exercer à l'orientation dans le monde en train d'être décrit en recourant aux savoirs présentés dans l'encart grammatical.

Notre proposition de matériel, enfin, revisite l'idée d'une "perspective incarnée " (Tomasello, 2005), en se penchant sur les éléments de l'énoncé dans leur ordre syntaxique. On s'est inscrit dans le cadre d'une linguistique dite énactive : le langage coordonne des actes de pensée émergeant avec la parole et ajuste les positions épistémiques des participants (il y a, de l'extérieur, échange d'informations) (Bottineau, 2010). En pédagogie de langue étrangère, l'approche énactive est sensible aux effets proprioceptifs de la parole et aux états affectifs qu'elle suscite (Aden, 2017 ; Lapaire, 2016). Notre angle d'attaque a visé la différence dans la morphosyntaxe des énoncés au passé composé et à l'imparfait. La valeur aspectuelle du passé composé, a-t-on suggéré, peut s'enseigner en tenant compte de la disposition d'esprit particulière dans laquelle le paradigme grammatical de la possession avec "avoir " met le sujet : "possesseur " d'un accompli exhibé dans l'espace de l'énonciation. Quant à l'imparfait (et au plus que parfait), on a exploité l'identité des dynamiques perceptuelles (Bottineau, 2014) avec le présent cursif (et l'accompli du présent). Le matériel comporte des visuels qui, en corrélation avec ces notions, sont censés aider à l'organisation temporelle du récit en un arrière-plan (simultanéité) et un premier plan (succession) (Reinhart, 1984). On suggère enfin des paraphrases pédagogiques accompagnant l'utilisation du matériel pendant les activités d'apprentissages (on a donné l'exemple d'un récit d'images).

73 Ce matériel ne pallie pas toutes les difficultés. Il est en cours d'expérimentation, avec des publics endolingue puis exolingue, et sera sans doute modifié. Mais on cherche dans sa conception à prendre en compte l'activité des apprenants lors de son utilisation, et les besoins changeants que son utilisation cause à mesure que les apprenants 
progressent dans l'activité d'apprentissage (fondamentalement, un récit). En l'occurrence, on avait pensé, dans des tentatives antérieures, que le choix du point de vue sur le procès était une composante suffisante de ce savoir faire, en donnant à la notion une importance considérable dans la conception du matériel (à l'instar, par exemple, des auteures de Libre Échange). On pense à présent que le point de vue n'est pas un moyen, ou bien qu'il est un moyen identique au but. L'organisation narrative en une trame de premier plan et en des circonstances à l'arrière-plan sert au maintien constant de cette perspective parce qu'elle situe le locuteur et l'interlocuteur dans le (leur) monde. Là est, nous semble-t-il, la clé du développement aux niveaux initiaux de la maitrise du récit dans l'apprentissage de langue étrangère.

\section{BIBLIOGRAPHIE}

Achard, Michel. 2008. Teaching construals : Cognitive pedagogical grammar. In Robinson P. \& Ellis N. C. (Eds.), Handbook of cognitive linguistics and second language acquisition (432-455). New York / London : Routledge.

Aden, Joëlle. 2017. Langues et langage dans un paradigme énactif. Recherches en didactique des langues et des cultures [En ligne], 14-1, consulté le 30 avril 2019. URL: http:// journals.openedition.org/rdlc/1085; DOI: 10.4000/rdlc.1085

Andersen, Roger W. \& Shirai, Yasuhiro. 1994. Discourse motivations for some cognitive acquisition principles. Studies in second language acquisition 16, 133-156.

Auchlin, Antoine. 2017. Prosodie, expérienciation, énaction. Intellectica 2, 68, 99-122.

Beacco, Jean-Claude. 2010. La didactique de la grammaire dans l'enseignement du français et des

langues. Paris : Éditions Didier.

Benveniste, Émile. 1974. Problèmes de linguistique générale, vol. 2. Paris : Gallimard.

Bergström, Anna. 1997. L'influence des distinctions aspectuelles sur l'acquisition des temps en français langue étrangère. Acquisition et interaction en langue étrangère, 9 [en ligne].URL : https:// journals.openedition.org/aile/1315, consulté le 10 septembre 2019.

Besse, Henri. 2005. De la nécessité présente d'une métalinguistique contrastive. In Mochet, M.-A. et al. (coord.), Plurilinguisme et apprentissage. Mélanges Daniel Coste, 71-87. Lyon : ENS Éditions.

Bottineau, Didier. 2010. Language and enaction. In Stewart J., Gapenne O. \& Di Paolo E. A. (eds.), Enaction. Toward a new paradigm for cognitive science, 267-306. Cambridge (MA) / London : The MIT Press.

Bottineau, Didier. 2014. Grammaire énactive et didactique du FLE. In Martinot, C. \& Pégaz Paquet A. (Éds.), Innovations didactiques en français langue étrangère, 185-200. Paris : CRL.

Courtillon, Janine. 1985. Pour une grammaire notionnelle. Langue française 68, 32-47

Courtillon, Janine. 2001. La mise en œuvre de la « grammaire du sens » dans l'approche communicative. Éla. Études de linguistique appliquée 2, 122, 153-164. 
Dansereau, Diane. 1987. A Discussion of techniques used in the teaching of the passé composé/ imparfait distinction in French. French Review 61, 33-38.

Gosselin, Laurent. 2005. Temporalité et modalité. Bruxelles : De Boeck / Duculot.

Gündüz, Marie-Pierre. 2005. Articulation problématique des descriptions grammaticales et des pseudo-compétences chez les apprenants de FLE. L'exemple des temps du passé. Acquisition et interaction en langue étrangère 23 [en ligne]. URL : https://journals.openedition.org/aile/1708, consulté le 10 septembre 2019.

Judge, Anne. 2002. Écarts entre manuels et réalité : un problème pour l'enseignement des temps du passé à des étudiants d'un niveau avancé. In Labeau, E. \& Larrivée, P. (Dir.), Les temps du passé français et leur enseignement, 135-156. Amsterdam / New York : Rodopi.

Labeau, Emmanuelle. 2002. L'unité de l'imparfait : vues théoriques et perspectives pour les apprenants du français langue étrangère. Travaux de linguistique 2, 45. 157-184.

Lapaire, Jean-Rémi. 2016. The choreography of time : metaphor, gesture and construal. In Gabriel, R. \& Pelosi, A. C. (Eds.), Linguagem e cognição : emergência e produção de sentidos, 217-234. Florianópolis : Insular.

Merleau-Ponty, Maurice. 2016. Phénoménologie de la perception. Paris : Gallimard. [1945]

Mourelatos, Alexander P. D. 1978. Events, processes, and states. Linguistics and philosophy 2, 415-434.

Poirier, Marine. 2017. Esquisse des principes d'une chronosignifiance. Signifiances (Signifying), 1(3), 41-66.

Pottier, Bernard. 2001. Représentations mentales et catégorisations linguistiques. Louvain / Paris : Éditions Peeters.

Puren, Christian. 2001. Représentations de l'enseignement apprentissage de la grammaire en didactique des langues. Éla. Études de linguistique appliquée 2, 122, 135-141.

Reinhart, Tanya. 1984. Principles of gestalt perception in the temporal organization of narrative texts. Linguistics 22, 779-809.

Renaud, Francis. 2002. Est-il possible d'effectuer une classification des types de situations vendleriennes par des tests syntaxiques? L'Information grammaticale 95, 23-26.

Salins (de), Geneviève-Dominique. 1996. Grammaire pour l'enseignement / apprentissage du FLE. Paris: Didier / Hatier.

Shirai, Yasuhiro. 2009. Temporality in first and second language acquisition. In Klein, W. \& Li, P. (Eds.), The expression of time, 167-194. Berlin / New York: Mouton de Gruyter.

Shirai, Yasuhiro. 2016.The acquisition of linguistic categories in second language acquisition: A functionalist approach. In Kabata, K. \& Toratoni, K. (Eds.), Cognitive-functional approaches to the study of Japanese as a second language, 13-32. Boston / Berlin: Walter de Gruyter.

Shirai, Yasuhiro \& Andersen, Roger W. 1995. The acquisition of tense-aspect morphology: A prototype account. Language 71/4, 743-762.

Tomasello, Michael. 2005. Constructing a language. A usage-based theory of language acquisition. Cambridge (MA) / London : Harvard University Press. [2003]

Véronique, Daniel (Dir). 2009. L'acquisition de la grammaire en français, langue étrangère. Paris : Didier. 
Victorri, Bernard. 1999. Le sens grammatical. Langages, 136, 85-015.

Vigner, Gérard. 2004. La grammaire en FLE. Paris : Hachette.

Weinrich, Harald. 1973. Le temps. Le récit et le commentaire. Paris : Seuil. [1964]

Whatley, Melissa. 2011. L'enseignement de la distinction passé composé/imparfait aux apprenants anglophones de FLE : un test de deux explications dans la salle de classe. In Neveu F., Muni Toke V., Durand J., Klingler T., Mondada L., Prévost S. (Éds.), Congrès Mondial de Linguistique Française - CMLF 2010, 645-655. Paris : Institut de Linguistique Française

\section{Manuels dont des extraits sont cités}

Carenzi-Vialaneix Cristelle, Metton, Catherine, Nachon, Annabelle \& Nugue, Fabienne. 2010. À propos. A2. Livre de l'élève. Monts : PUG.

Cocton, Marie-Noëlle, De Oliveira, Anouchka, Dintilhac, Anneline, Dupleix, Dorothée \& Ripaud, Delphine. 2014. Saison 2. Méthode de français. Paris : Didier.

Courtillon, Janine\& de Salins, Geneviève-Dominique. 2003. Libre Échange. Méthode de français. Niveau 1. Livre de l'élève. Paris : Hatier / Didier.

Denyer, Monique, Garmendia, Agustin, Royer, Corinne \& Lions-Olivieri, Marie-Laure. 2010. Version originale 2. Méthode de français. Livre de l'élève. Paris : difusión / ÉDML.

Di Giura, Marcella \& Beacco, Jean-Claude. 2015. Alors. Méthode de français fondée sur l'approche par compétences. Niveau A2 du CECR. Paris : Didier.

Heu, Élodie, Abou-Samra, Myriam, Braud, Céline, Brunelle, Michèle, Perrad, Marion \& Pinson, Cécile. 2016. Édito. Méthode de français. Niveau A2. Paris : Didier.

Lopes, Marie-José, Le Bougnec Jean-Thierry, Lewis, Guy \& Mous, Nelly. 2014. Totem 2. Méthode de français. Guide pédagogique. Paris : Hachette.

Poisson-Quinton, Sylvie \& Mimran, Reine. 2011. Amical 2. Méthode de français. Paris : CLE International / Sejer.

\section{NOTES}

1. Mentionner la variété de la terminologie pour l'aspect est un lieu commun. Pour distinguer nos choix terminologiques sur le seul plan de l'analyse intralinguistique, on se référera à Gosselin (2005). Cet auteur pose un intervalle correspondant à la "fenêtre de monstration " (Victorri, 1999) sur le procès. La correspondance des bornes de cet intervalle avec celles du procès définit l'aspect « aoristique » ou « perfectif ». L'aspect « imperfectif », « inaccompli » ou « sécant » vient de ce que les bornes de l'intervalle sont à l'intérieur des bornes du procès. Pour l'aspect « accompli », il s'agit d'une vision sécante sur le résultat du procès, c'est-à-dire que la fenêtre de monstration est à droite de la borne droite du procès. Enfin, un aspect « prospectif » correspond à la position de l'intervalle à gauche du procès (Gosselin, $2005: 36$ ). En ce qui nous concerne, nous soulignerons l'importance de la syntaxe pour la distinction d'aspect dans notre proposition. Pour cette raison, nous choisissons les termes «accompli / inaccompli» qui peuvent mieux correspondre à cette dimension.

2. Notons ici que tout comme la fréquence, ni la durée ni le moment ne sont des propriétés du verbe conjugué. Il est correct de dire que les expressions de fréquence sont utilisées préférentiellement avec un temps imperfectif (voir note suivante). Pourtant, dans les présentations grammaticales, cette propriété apparaittra à l'imparfait à travers les notions d'« habitude révolue », « habitude dans le passé », etc. 
3. Chez l'enfant, la perception de l'itération de procès ponctuels (exemple : to cough) est perçue très vite comme atélique, pouvant être décrite dans son cours (Shirai \& Andersen, 1995). Selon nous, ce pourrait être un patron (pattern) de la convergence des marqueurs temporels de fréquence et des temps imperfectifs, par exemple, Le dimanche, j'allais à l'église (exemple dans Dansereau (1987).

4. Mais Courtillon (1985) s'étonne de ce choix d'exemple apparemment déjà très commun: «Pourquoi pas : «Quand je suis entré, elle a fermé (ouvert) son livre » ou encore « Quand je suis entrée, elle avait déjà lu tout le livre "? On ne voit vraiment pas pourquoi la simultanéité dans le passé induirait nécessairement une opposition aspectuelle de type "action en cours d'accomplissement - action accomplie »? » (p. 37) Ce que Dansereau veut mettre en place semble simplement être le rôle des deux temps dans l'organisation temporelle en deux plans, avec les procès à l'imparfait en arrière-plan.

5. L'auteure fait référence aux travaux qui exploitent l'opposition deixis / anaphore pour la description des temps verbaux du passé. Pour comparaison, l'intervalle de référence de Gosselin (2005, voir note 1) - et non pas le point - permet de décrire un ancrage (déictique ou anaphorique) dans le contexte linguistique (ou extralinguistique) mais aussi de définir les relations aspectuelles suivant la coupe que l'intervalle opère sur l'axe temporel.

6. Liste des 17 manuels : À propos A2 (2010), Agenda 2 (2011), Alors 2 (2015), Alter Ego 2 (2006), Alter Ego + A2 (2012), Amical 2 (2011), Édito A2 (2016), Entre Nous 2 (2015), Le Nouveau Taxi 2 (2009), Nickel 2 (2015), Nouveau Rond-Point 2 (2011), Saison 2 (2014), Tendances 2 (2016), Totem 2 (2012), Tout va bien! 2 (2005), Version Originale 2 (2010), Zénith 2 (2012).

7. Liste des 5 manuels : À propos A2 (2010), Alors 2 (2015), Édito A2 (2016), Totem 2 (2012), Version Originale 2 (2010).

8. En fait, sur les 17 présentations grammaticales analysées, seule une emploie ces termes: «L'imparfait sert à :

- décrire les circonstances d'un événement passé et à indiquer un déroulement (long ou bref) des événements de second plan. Le passé composé, à l'opposé, note des événement, situés dans le passé de premier plan.

Il faisait beau. J'étais sur la plage quand j'ai vu...

- exprimer l'habitude, dans le passé.

Tous les matins, Jean lui apportait des croissants chauds. Mais un jour il a oublié... (Alors A2, p. 147)

9. Excepté Entre Nous 2, de la même maison d'édition.

10. Dans un manuel, une activité vraiment axée sur l'alternance des deux temps n'a pu être trouvée.

11. La transposition au passé d'un texte au présent est, on l'a vu, un exercice typique du choix des temps dans les activités de pratique de notre corpus. Cet exercice fait intervenir, notamment, l'aspect lexical. En se basant sur Gosselin (2005 : 209), on rappellera que dans le récit au présent de narration, en général, les procès téliques se succèderont au premier plan et les procès atéliques seront simultanés à l'arrière-plan, indiquant les causes, les circonstances, etc.

12. Pour une phase de réflexion après une activité de repérage entre " action » et "situations ", un des guides pédagogiques des manuels du corpus conseille en ces termes : «[i]nsister sur le fait qu'à l'imparfait rien ne change. Il ne se passe rien. » (Totem 2. Guide pédagogique, p. 61)

13. Un évaluateur se demande si on ne peut pas affirmer du passé composé, dans sa valeur d'antérieur au présent, qu'il est également «un présent qui n'est ni ici ni maintenant ». Nos propositions de paraphrases pédagogiques visent à instaurer le contraste modal entre le présent et l'imparfait, contraste qui n'existe pas entre le présent et le passé composé (ce dernier temps étant de toute façon un présent du point de vue morphosyntaxique). Le passé composé n'est pas un temps du virtuel, comme l'est l'imparfait (cf. notamment ses emplois contrefactuels dans la protase avec « si », parfois dans l'apodose à la place du conditionnel passé et pour l'imminence contrecarrée). L'expérience de pensée suggérée dans les paraphrases s'appuie sur la perception 
du procès en cours au moment de l'énonciation de ces paraphrases, typiquement « être (ici, dans la classe) ». Cela revient à essayer d'attirer l'attention sur l'expérience du passage du temps qui nous situe dans le cours de ce procès, c'est-à-dire avant son aboutissement. En d'autres termes, cette phase de l'instruction exploite la similitude aspectuelle du présent (pour un de ses emplois) et de l'imparfait dans leur valeur identique d'inaccompli. Ces paraphrases fonctionnent en opposition avec la notion d'accompli telle que celle-ci est représentée iconiquement dans notre matériel comme une forme fermée, littéralement un fait montré «ici et maintenant» à l'interlocuteur. Le passé composé possède intrinsèquement ce trait de factualité marquant l'énonciation, même dans sa valeur dite d'antérieur au présent.

14. Un évaluateur observe avec raison que la préhension de l'accompli par la main pourrait être représentée du côté du résultat, c'est-à-dire de la borne finale $\mathrm{B}$.

\section{RÉSUMÉS}

Cet article a pour objet la conception de matériel pédagogique pour l'apprentissage endolingue de la relation entre le passé composé et l'imparfait dans le récit en FLE. Faut-il privilégier le choix d'une visée sur le procès ou bien montrer que la distribution des temps s'effectue à partir d'indices contextuels qu'il faut repérer? La première position a été représentée, par exemple, dans le manuel Libre Échange (2001), mais délaissée par la suite (Gündüz, 2005). Notre propre analyse de 17 manuels publiés entre 2006 et 2016 confirme la tendance. On constate en outre qu'il n'y a aucun exemple d'un même procès sous les deux aspects accompli et inaccompli (j'ai voulu/je voulais), comme si cette option avait été bannie. Toutefois, l'approche aspectuelle n'est pas toutà-fait absente : on considère le cas d'un manuel tendant à intégrer présence d'un sujet opérant des choix de visée et emplois normés des temps. Finalement, on propose un matériel qui exploite la syntaxe distincte des énoncés avec l'imparfait et le passé composé. Dans un cadre énactif (Bottineau, 2010, 2014), on envisage que l'énonciateur prend deux états différents, où il perçoit un présent imaginé, ou bien où il devient " possesseur » d'un accompli. On détaille la conception théorique, décrit des visuels extraits du matériel, et suggère une gestuelle pédagogique pour montrer comment cette approche peut soutenir l'apprentissage des temps du récit.

This article addresses the issue of pedagogical materials development for the learning of the relation between the compound past (passé composé) and the imperfect (imparfait) in narrative in French as a Foreign Language in endolingual context. Should the viewing choice on the situation be favored, or or should we teach that tenses are used based on contextual clues that need first to be identified? The first position was represented, for example, in the textbook Libre Échange (2001), but later on abandoned (Gündüz, 2005). Our own analysis of 17 textbooks published between 2006 and 2016 confirms this trend. Not one example has been found of the same situation viewed under both bounded and unbounded aspects (j'ai voulu / je voulais), as though this option had been banned. However, the aspectual approach is not completely absent, as demonstrates the case of one textbook that tends to acknowledge both the agency of a subject choosing a view on the situation and norms in the using of the two tenses. Finally, a different material is proposed, that exploits the syntax of utterances with the imparfait and the passé composé. In an enactive framework (Bottineau, 2010, 2014), it is envisaged that two states are being achieved: the perception of an imagined present, or the "possession" of a bounded event. A 
theoretical account is given, visuals from the material are described and pedagogical gesture suggested to show how this approach can be conducive to the learning of tenses in narrative.

INDEX

Mots-clés : Français Langue Étrangère, temps du passé, aspect, conception de matériel pédagogique, contexte endolingue, linguistique énactive.

Keywords : French as a Foreign Language, past tenses, aspect, pedagogical materials development, endolingual context, enactive linguistics.

\section{AUTEUR \\ LOÏC RENOUD}

Université d'okayama 